\title{
Excited States of $\mathrm{Pt}\left(\mathrm{PF}_{3}\right)_{4}$ and Their Role in Focused Electron-Beam Nanofabrication SUPPLEMENTARY INFORMATION
}

\author{
Matija Zlatar, ${ }^{*, \dagger}$ Michael Allan, ${ }^{*, \ddagger}$ and Juraj Fedor, \\ $\dagger$ Department of Chemistry, Institute of Chemistry, Technology and Metallurgy (IChTM), \\ University of Belgrade, Njegoševa 12, P.O. Box 815, 11001 Belgrade, Serbia \\ $\ddagger$ Departement of Chemistry, University of Fribourg, Chemin du musée 9, 1700 Fribourg, \\ Switzerland \\ ФJ. Heyrovský Institute of Physical Chemistry v.v.i., The Czech Academy of Sciences, \\ Dolejškova 3, 18223 Prague, Czech Republic
}

E-mail: matijaz@chem.bg.ac.rs; michael.allan@unifr.ch; juraj.fedor@jh-inst.cas.cz 
Table S1: Pt-P bond lengths $(\AA)$ and at different levels of theory and comparison with previously reported results.

\begin{tabular}{lcc|llll}
\multicolumn{3}{c|}{ Present results } & \multicolumn{4}{c}{ Previous results } \\
\hline \multirow{3}{*}{ Relativistic } & Non-relativistic & \multicolumn{3}{|c}{ Theory } & & Experiment \\
B3LYP & 2.222 & 2.332 & MP2 $^{1}$ & 2.239 & & \\
B3LYP-D3 & 2.279 & 2.457 & BP86 $^{2}$ & 2.276 & X-ray $^{1}$ & 2.224 \\
& 2.266 & 2.428 & MPW91PW91 $^{2}$ & 2.255 & El. diffr $^{3}$ & 2.229
\end{tabular}

\section{Comparison of calculated molecular properties with ex-}

\section{perimental data}

In order to test the performance of theoretical methods we have calculated the basic properties of $\mathrm{Pt}\left(\mathrm{PF}_{3}\right)_{4}$ and compared them to available experimental data. Table $\mathrm{S} 1$ shows the comparison for the Pt-P bond length. The experimental values are $2.229 \AA$ as determined by electron diffraction and $2.224 \AA$ as determined by X-ray scattering. In the Table S1 the results of previous calculation ${ }^{1}$ at MP2 level with relativistic corrected core potentials are included as well. The best agreement with experimental bond lengths have been achieved with scalar relativistic (SR) LDA/TZP level of theory. SR-B3LYP/TZP and SR-B3LYP-D3/TZP give slightly longer Pt-P bond lengths. Inclusion of relativistic corrections is indispensable for reasonable agreement with the experiment.

Table S2 shows the calculated vibrational frequencies and their comparison with experimental data. SR calculations show better agreement with experimentally determined frequencies. Non-relativistic (NR) calculations give lower frequencies, corroborating longer bond lengths, Table S1.

\section{Bonding analysis}

Results for the energy decomposition analysis of $\mathrm{Pt}\left(\mathrm{PF}_{3}\right)_{4}$ at different levels of theory are summarized in Table S3. This nature of bonding, in principle, does not change at different level of theory, however interaction energy between two fragments is significantly lower if 
Table S2: Selected vibrational frequencies of $\mathrm{Pt}\left(\mathrm{PF}_{3}\right)_{4} \quad(\mathrm{meV})$ at different levels of theory and comparison with previously reported results.

\begin{tabular}{l|cccccc|cc}
\multirow{2}{*}{ Description } & \multicolumn{5}{|c|}{ Present results } & Previous work \\
& LDA & B3LYP & B3LYP-D3 & LDA & B3LYP & B3LYP-D3 & Exp. $^{4}$ & Exp. $^{5}$ \\
\hline P-F symm str & 109 & 107 & 108 & 108 & 106 & 106 & 119 & 113 \\
P-F asymm str & 104 & 102 & 103 & 103 & 102 & 102 & 106 & 107 \\
$\mathrm{PF}_{3}$ symm def & 60 & 60 & 60 & 57 & 57 & 57 & 68 & 65 \\
$\mathrm{PF}_{3}$ asymm def & 43 & 44 & 44 & 41 & 42 & 42 & 41 & 48 \\
$\mathrm{PF}_{3}$ rock & 26 & 24 & 25 & 19 & 14 & 16 & 35 & 35 \\
$\mathrm{Pt}_{\text {-P asymm str }}$ & 28 & 26 & 27 & 23 & 18 & 19 & 27 & 26 \\
$\mathrm{PtP}_{4}$ def & 6 & 7 & 6 & 3 & 4 & 4 & 6 & 6
\end{tabular}

NR calculations are employed. This is in accordance with longer $\mathrm{Pt}-\mathrm{P}$ equilibrium bond lengths in non-relativistic situation, Table S1.

Table S3: Energy decomposition analysis of $\mathrm{Pt}\left(\mathrm{PF}_{3}\right)_{3}--\mathrm{PF}_{3}$ (energy components given in $\mathrm{eV}$; values in parentheses give the percentage of the total attractive interactions: $\left.E_{\text {elst }}+E_{\text {orb }}+E_{\text {disp }}\right)$

\begin{tabular}{l|ll|ll} 
& \multicolumn{2}{|c|}{ Relativistic } & \multicolumn{2}{|c}{ Non-relativistic } \\
& B3LYP & B3LYP-D3 & B3LYP & B3LYP-D3 \\
\hline$E_{\text {def }}\left(\mathrm{Pt}_{\left.\left(\mathrm{PF}_{3}\right)_{3}\right)}\right)$ & 0.7183 & 0.6812 & 0.4341 & 0.4113 \\
$E_{\text {def }}\left(\mathrm{PF}_{3}\right)$ & 0.0128 & 0.0153 & 0.0057 & 0.0069 \\
$E_{\text {prep }}$ & 0.7311 & 0.6965 & 0.4398 & 0.4182 \\
\hline$E_{\text {Pauli }}$ & 7.3848 & 7.7207 & 4.1519 & 4.5791 \\
$E_{\text {elst }}$ & $-5.8000(67 \%)$ & $-6.0468(65 \%)$ & $-3.3163(67 \%)$ & $-3.6266(64 \%)$ \\
$E_{\text {orb }}$ & $-2.8337(33 \%)$ & $-2.9196(31 \%)$ & $-1.6200(33 \%)$ & $-1.7259(31 \%)$ \\
$E_{\text {disp }}$ & - & $-0.3567(4 \%)$ & - & $-0.3001(5 \%)$ \\
$E_{\text {int }}$ & -1.2488 & -1.6025 & -0.7844 & -1.0735 \\
\hline$B D E$ & 0.5178 & 0.9060 & 0.3446 & 0.6553
\end{tabular}

\section{Excited states}

Tables S4, S5, S6 list the calculated excited states, their energies and oscillator strengths. The excitation energies were calculated at the SR-LDA/TZP ground state geometry using the SR$\mathrm{SAOP} / \mathrm{TZP}$ method (see Figure 1 in the main text). Ground electronic state of $\mathrm{Pt}\left(\mathrm{PF}_{3}\right)_{4}$ is ${ }^{1} A_{1}$, with electronic configuration: ...(7e $)^{4}\left(6 t_{1}\right)^{6}\left(20 t_{2}\right)^{6}(8 e)^{4}\left(7 t_{1}\right)^{6}\left(14 a_{1}\right)^{2}\left(8 t_{1}\right)^{6}\left(1 a_{2}\right)^{2}\left(21 t_{2}\right)^{6}(9 e)^{4}$ 
$\left(22 t_{2}\right)^{6}\left(23 t_{2}\right)^{0}(10 e)^{0}\left(9 t_{1}\right)^{0}\left(15 a_{1}\right)^{0}\left(16 a_{1}\right)^{0}\left(24 t_{2}\right)^{0}\left(25 t_{2}\right)^{0}(11 e)^{0}\left(17 a_{1}\right)^{0}\left(26 t_{2}\right)^{0}\left(10 t_{1}\right)^{0}\left(27 t_{2}\right)^{0} \ldots$ Description of electronic states by TDDFT is given by linear combination of single excitations, as given in Tables $\mathrm{S} 4, \mathrm{~S} 5$ and $\mathrm{S} 6$.

Dipole allowed transitions are to the excited ${ }^{1} T_{2}$ states, Table S4. Spin allowed, dipole forbidden transitions are given in Table S5, while singlet-triplet transitions are collected in Table S6. 
Table S4: TDDFT (SR-SAOP/TZP//SR-LDA/TZP) spin and dipole allowed transitions, the corresponding oscillator strenghts (f), and description of the dominant one-electron excitations (contributions greater than $10 \%$ of $\mathrm{Pt}\left(\mathrm{PF}_{3}\right)_{4}$

\begin{tabular}{|c|c|c|c|c|c|c|c|}
\hline $\mathrm{E}(\mathrm{eV})$ & $\mathrm{f}$ & Assignment & $\%$ & $\mathrm{E}(\mathrm{eV})$ & $\mathrm{f}$ & Assignment & $\%$ \\
\hline \multirow[t]{2}{*}{5.885} & $0.44364 \mathrm{E}-01$ & $22 t_{2} \rightarrow 23 t_{2}$ & 77.11 & 10.599 & $0.37307 \mathrm{E}-01$ & $22 t_{2} \rightarrow 17 a_{1}$ & 69.66 \\
\hline & & $22 t_{2} \rightarrow 10 e$ & 18.55 & & & $8 t_{1} \rightarrow 10 e$ & 10.94 \\
\hline \multirow[t]{3}{*}{6.525} & 0.12539 & $22 t_{2} \rightarrow 10 e$ & 70.30 & 10.648 & $0.11706 \mathrm{E}-01$ & $8 t_{1} \rightarrow 10 e$ & 76.79 \\
\hline & & $22 t_{2} \rightarrow 15 a_{1}$ & 14.28 & 10.779 & 0.43323E-02 & $22 t_{2} \rightarrow 26 t_{2}$ & 85.41 \\
\hline & & $22 t_{2} \rightarrow 23 t_{2}$ & 11.44 & 10.937 & $0.98605 \mathrm{E}-02$ & $7 t_{1} \rightarrow 10 e$ & 90.51 \\
\hline \multirow[t]{2}{*}{6.904} & $0.94420 \mathrm{E}-01$ & $22 t_{2} \rightarrow 15 a_{1}$ & 54.71 & 10.971 & $0.11875 \mathrm{E}-02$ & $9 e \rightarrow 24 t_{2}$ & 94.55 \\
\hline & & $22 t_{2} \rightarrow 9 t_{1}$ & 37.68 & 11.058 & 0.81334E-04 & $20 t_{2} \rightarrow 10 e$ & 91.94 \\
\hline \multirow[t]{2}{*}{7.236} & 0.28466 & $22 t_{2} \rightarrow 9 t_{1}$ & 54.09 & 11.094 & $0.26430 \mathrm{E}-03$ & $1 a_{2} \rightarrow 9 t_{1}$ & 47.15 \\
\hline & & $22 t_{2} \rightarrow 15 a_{1}$ & 26.22 & & & $8 t_{1} \rightarrow 9 t_{1}$ & 49.17 \\
\hline \multirow[t]{2}{*}{8.246} & $0.17888 \mathrm{E}-01$ & $22 t_{2} \rightarrow 16 a_{1}$ & 46.85 & 11.107 & 0.72610E-02 & $21 t_{2} \rightarrow 9 t_{1}$ & 51.98 \\
\hline & & $22 t_{2} \rightarrow 24 t_{2}$ & 46.27 & & & $8 t_{1} \rightarrow 9 t_{1}$ & 19.75 \\
\hline \multirow[t]{2}{*}{8.350} & $0.73354 \mathrm{E}-03$ & $22 t_{2} \rightarrow 16 a_{1}$ & 45.25 & 11.190 & $0.50058 \mathrm{E}-02$ & $21 t_{2} \rightarrow 15 a_{1}$ & 22.98 \\
\hline & & $22 t_{2} \rightarrow 24 t_{2}$ & 50.75 & & & $1 a_{2} \rightarrow 9 t_{1}$ & 18.08 \\
\hline 8.568 & 0.12845 & $9 e \rightarrow 23 t_{2}$ & 85.91 & & & $22 t_{2} \rightarrow 10 t_{1}$ & 34.20 \\
\hline 9.551 & 0.18121 & $9 e \rightarrow 9 t_{1}$ & 85.25 & & & $8 t_{1} \rightarrow 9 t_{1}$ & 14.11 \\
\hline 9.655 & $0.31007 \mathrm{E}-01$ & $22 t_{2} \rightarrow 25 t_{2}$ & 98.80 & 11.196 & 0.10471 & $21 t_{2} \rightarrow 15 a_{1}$ & 36.79 \\
\hline \multirow[t]{2}{*}{10.056} & $0.78400 \mathrm{E}-01$ & $21 t_{2} \rightarrow 23 t_{2}$ & 76.32 & & & $22 t_{2} \rightarrow 10 t_{1}$ & 51.33 \\
\hline & & $8 t_{1} \rightarrow 23 t_{2}$ & 11.42 & 11.227 & $0.73014 \mathrm{E}-01$ & $1 a_{2} \rightarrow 9 t_{1}$ & 18.88 \\
\hline 10.110 & $0.52105 \mathrm{E}-01$ & $8 t_{1} \rightarrow 23 t_{2}$ & 84.16 & & & $21 t_{2} \rightarrow 15 a_{1}$ & 11.36 \\
\hline 10.224 & $0.38502 \mathrm{E}-01$ & $14 a_{1} \rightarrow 23 t_{2}$ & 82.79 & & & $21 t_{2} \rightarrow 9 t_{1}$ & 14.97 \\
\hline 10.409 & 0.79109E-02 & $7 t_{1} \rightarrow 23 t_{2}$ & 84.85 & & & $22 t_{2} \rightarrow 27 t_{2}$ & 13.76 \\
\hline \multirow[t]{2}{*}{10.468} & $0.44058 \mathrm{E}-02$ & $22 t_{2} \rightarrow 11 e$ & 77.17 & & & $22 t_{2} \rightarrow 10 t_{1}$ & 10.96 \\
\hline & & $8 e \rightarrow 23 t_{2}$ & 11.31 & & & $8 t_{1} \rightarrow 9 t_{1}$ & 10.80 \\
\hline \multirow[t]{2}{*}{10.506} & $0.24861 \mathrm{E}-02$ & $21 t_{2} \rightarrow 10 e$ & 45.37 & 11.391 & $0.15589 \mathrm{E}-02$ & $6 t_{1} \rightarrow 23 t_{2}$ & 86.73 \\
\hline & & $20 t_{2} \rightarrow 23 t_{2}$ & 35.93 & 11.416 & $0.54315 \mathrm{E}-04$ & $7 e \rightarrow 23 t_{2}$ & 39.26 \\
\hline \multirow[t]{3}{*}{10.530} & $0.36624 \mathrm{E}-03$ & $8 e \rightarrow 23 t_{2}$ & 46.26 & & & $19 t_{2} \rightarrow 23 t_{2}$ & 53.19 \\
\hline & & $21 t_{2} \rightarrow 10 e$ & 16.39 & 11.463 & $0.19230 \mathrm{E}-03$ & $7 t_{1} \rightarrow 9 t_{1}$ & 76.50 \\
\hline & & $20 t_{2} \rightarrow 23 t_{2}$ & 19.76 & & & $20 t_{2} \rightarrow 9 t_{1}$ & 13.41 \\
\hline \multirow[t]{3}{*}{10.538} & $0.10297 \mathrm{E}-01$ & $8 e \rightarrow 23 t_{2}$ & 38.55 & & & & \\
\hline & & $20 t_{2} \rightarrow 23 t_{2}$ & 28.47 & & & & \\
\hline & & $21 t_{2} \rightarrow 10 e$ & 16.48 & & & & \\
\hline
\end{tabular}

Table S5: TDDFT (SR-SAOP/TZP//SR-LDA/TZP) spin allowed, dipole forbidden transitions, their symmetry label, the corresponding oscillator strenghts (f), and description of the dominant one-electron excitations (contributions greater than $10 \%)$ of $\mathrm{Pt}\left(\mathrm{PF}_{3}\right)_{4}$

\begin{tabular}{llll}
\hline Symmetry & $\mathrm{E}(\mathrm{eV})$ & $\mathrm{f}$ & Assignment
\end{tabular}


Table S5: TDDFT (SR-SAOP/TZP//SR-LDA/TZP) dipole forbidden transitions

\begin{tabular}{|c|c|c|c|c|}
\hline Symmetry & $\mathrm{E}(\mathrm{eV})$ & $\mathrm{f}$ & Assignment & $\%$ \\
\hline \multirow[t]{22}{*}{$A_{1}$} & 5.9241 & 0.0000 & $22 t_{2} \rightarrow 23 t_{2}$ & 96.45 \\
\hline & 8.4208 & 0.0000 & $22 t_{2} \rightarrow 24 t_{2}$ & 94.98 \\
\hline & 9.3659 & 0.0000 & $9 e \rightarrow 10 e$ & 62.74 \\
\hline & & & $21 t_{2} \rightarrow 23 t_{2}$ & 33.85 \\
\hline & 9.6158 & 0.0000 & $22 t_{2} \rightarrow 25 t_{2}$ & 99.83 \\
\hline & 10.511 & 0.0000 & $20 t_{2} \rightarrow 23 t_{2}$ & 71.67 \\
\hline & & & $2 t_{2} \rightarrow 26 t_{2}$ & 12.01 \\
\hline & & & $21 t_{2} \rightarrow 23 t_{2}$ & 11.17 \\
\hline & 10.701 & 0.0000 & $22 t_{2} \rightarrow 26 t_{2}$ & 53.93 \\
\hline & & & $20 t_{2} \rightarrow 23 t_{2}$ & 20.74 \\
\hline & & & $21 t_{2} \rightarrow 23 t_{2}$ & 10.72 \\
\hline & 11.017 & 0.0000 & $8 e \rightarrow 10 e$ & 49.20 \\
\hline & & & $14 a_{1} \rightarrow 15 a_{1}$ & 20.64 \\
\hline & & & $22 t_{2} \rightarrow 26 t_{2}$ & 18.13 \\
\hline & 11.099 & 0.0000 & $8 t_{1} \rightarrow 9 t_{1}$ & 99.97 \\
\hline & 11.152 & 0.0000 & $14 a_{1} \rightarrow 15 a_{1}$ & 43.48 \\
\hline & & & $8 e \rightarrow 10 e$ & 34.14 \\
\hline & 11.420 & 0.0000 & $22 t_{2} \rightarrow 27 t_{2}$ & 57.34 \\
\hline & & & $19 t_{2} \rightarrow 23 t_{2}$ & 34.63 \\
\hline & 11.436 & 0.0000 & $19 t_{2} \rightarrow 23 t_{2}$ & 63.13 \\
\hline & & & $22 t_{2} \rightarrow 27 t_{2}$ & 28.00 \\
\hline & 11.568 & 0.0000 & $7 t_{1} \rightarrow 9 t_{1}$ & 68.78 \\
\hline \multirow[t]{2}{*}{$A_{2}$} & 6.6127 & 0.0000 & $22 t_{2} \rightarrow 9 t_{1}$ & 100.00 \\
\hline & 8.7871 & 0.0000 & $9 e \rightarrow 10 e$ & 100.00 \\
\hline
\end{tabular}


Table S5: TDDFT (SR-SAOP/TZP//SR-LDA/TZP) dipole forbidden transitions

\begin{tabular}{|c|c|c|c|c|}
\hline Symmetry & $\mathrm{E}(\mathrm{eV})$ & $\mathrm{f}$ & Assignment & $\%$ \\
\hline & 10.068 & 0.0000 & $8 t_{1} \rightarrow 23 t_{2}$ & 99.70 \\
\hline & 10.369 & 0.0000 & $7 t_{1} \rightarrow 23 t_{2}$ & 99.76 \\
\hline & 10.929 & 0.0000 & $21 t_{2} \rightarrow 9 t_{1}$ & 99.83 \\
\hline & 11.004 & 0.0000 & $8 e \rightarrow 10 e$ & 99.37 \\
\hline & 11.187 & 0.0000 & $22 t_{2} \rightarrow 10 t_{1}$ & 99.92 \\
\hline & 11.234 & 0.0000 & $1 a_{2} \rightarrow 15 a_{1}$ & 97.16 \\
\hline & 11.393 & 0.0000 & $6 t_{1} \rightarrow 23 t_{2}$ & 97.48 \\
\hline$E$ & 5.8437 & 0.0000 & $22 t_{2} \rightarrow 23 t_{2}$ & 96.36 \\
\hline & 6.9036 & 0.0000 & $22 t_{2} \rightarrow 9 t_{1}$ & 95.67 \\
\hline & 8.2597 & 0.0000 & $22 t_{2} \rightarrow 24 t_{2}$ & 99.97 \\
\hline & 9.0108 & 0.0000 & $9 e \rightarrow 10 e$ & 97.08 \\
\hline & 9.5683 & 0.0000 & $9 e \rightarrow 15 a_{1}$ & 91.34 \\
\hline & 9.6728 & 0.0000 & $22 t_{2} \rightarrow 25 t_{2}$ & 93.37 \\
\hline & 10.024 & 0.0000 & $21 t_{2} \rightarrow 23 t_{2}$ & 84.99 \\
\hline & & & $8 t_{1} \rightarrow 23 t_{2}$ & 10.48 \\
\hline & 10.116 & 0.0000 & $8 t_{1} \rightarrow 23 t_{2}$ & 85.94 \\
\hline & 10.376 & 0.0000 & $7 t_{1} \rightarrow 23 t_{2}$ & 98.77 \\
\hline & 10.524 & 0.0000 & $20 t_{2} \rightarrow 23 t_{2}$ & 95.51 \\
\hline & 10.620 & 0.0000 & $1 a_{2} \rightarrow 10 e$ & 80.71 \\
\hline & & & $14 a_{1} \rightarrow 10 e$ & 13.60 \\
\hline & 10.682 & 0.0000 & $14 a_{1} \rightarrow 10 e$ & 49.71 \\
\hline & & & $22 t_{2} \rightarrow 26 t_{2}$ & 37.98 \\
\hline & 10.725 & 0.0000 & $22 t_{2} \rightarrow 26 t_{2}$ & 55.65 \\
\hline & & & $14 a_{1} \rightarrow 10 e$ & 23.72 \\
\hline
\end{tabular}


Table S5: TDDFT (SR-SAOP/TZP//SR-LDA/TZP) dipole forbidden transitions

\begin{tabular}{|c|c|c|c|c|}
\hline \multirow[t]{12}{*}{ Symmetry } & $\mathrm{E}(\mathrm{eV})$ & $\mathrm{f}$ & Assignment & $\%$ \\
\hline & 10.853 & 0.0000 & $9 e \rightarrow 16 a_{1}$ & 90.16 \\
\hline & 11.026 & 0.0000 & $8 e \rightarrow 10 e$ & 96.69 \\
\hline & 11.100 & 0.0000 & $21 t_{2} \rightarrow 9 t_{1}$ & 65.26 \\
\hline & & & $8 t_{1} \rightarrow 9 t_{1}$ & 20.21 \\
\hline & 11.188 & 0.0000 & $22 t_{2} \rightarrow 10 t_{1}$ & 85.27 \\
\hline & & & $8 t_{1} \rightarrow 9 t_{1}$ & 13.30 \\
\hline & 11.234 & 0.0000 & $8 t_{1} \rightarrow 9 t_{1}$ & 52.41 \\
\hline & & & $19 t_{2} \rightarrow 23 t_{2}$ & 13.97 \\
\hline & & & $21 t_{2} \rightarrow 9 t_{1}$ & 14.75 \\
\hline & 11.389 & 0.0000 & $6 t_{1} \rightarrow 23 t_{2}$ & 89.09 \\
\hline & 11.408 & 0.0000 & $22 t_{2} \rightarrow 27 t_{2}$ & 92.56 \\
\hline \multirow[t]{13}{*}{$T_{1}$} & 5.7151 & $0.20106 \mathrm{e}-33$ & $22 t_{2} \rightarrow 23 t_{2}$ & 92.97 \\
\hline & 6.1971 & $0.33217 \mathrm{e}-32$ & $22 t_{2} \rightarrow 10 e$ & 92.13 \\
\hline & 6.7380 & $0.83888 \mathrm{e}-34$ & $22 t_{2} \rightarrow 9 t_{1}$ & 95.54 \\
\hline & 8.2606 & $0.49880 \mathrm{e}-35$ & $22 t_{2} \rightarrow 24 t_{2}$ & 99.74 \\
\hline & 8.3061 & $0.12464 \mathrm{e}-32$ & $9 e \rightarrow 23 t_{2}$ & 99.60 \\
\hline & 9.3172 & $0.14140 \mathrm{e}-32$ & $9 e \rightarrow 9 t_{1}$ & 99.83 \\
\hline & 9.6414 & $0.18301 \mathrm{e}-35$ & $22 t_{2} \rightarrow 25 t_{2}$ & 98.76 \\
\hline & 9.9405 & $0.18501 \mathrm{e}-36$ & $21 t_{2} \rightarrow 23 t_{2}$ & 94.83 \\
\hline & 10.062 & $0.62807 \mathrm{e}-36$ & $1 a_{2} \rightarrow 23 t_{2}$ & 49.36 \\
\hline & & & $8 t_{1} \rightarrow 23 t_{2}$ & 50.25 \\
\hline & 10.098 & $0.10198 \mathrm{e}-35$ & $1 a_{2} \rightarrow 23 t_{2}$ & 47.30 \\
\hline & & & $8 t_{1} \rightarrow 23 t_{2}$ & 48.02 \\
\hline & 10.390 & $0.39794 \mathrm{e}-37$ & $7 t_{1} \rightarrow 23 t_{2}$ & 98.19 \\
\hline
\end{tabular}


Table S5: TDDFT (SR-SAOP/TZP//SR-LDA/TZP) dipole forbidden transitions

\begin{tabular}{|c|c|c|c|c|}
\hline Symmetry & $\mathrm{E}(\mathrm{eV})$ & $\mathrm{f}$ & Assignment & $\%$ \\
\hline & \multirow[t]{2}{*}{10.448} & \multirow[t]{2}{*}{$0.22650 \mathrm{e}-34$} & $21 t_{2} \rightarrow 10 e$ & 63.25 \\
\hline & & & $22 t_{2} \rightarrow 11 e$ & 28.77 \\
\hline & \multirow[t]{2}{*}{10.473} & \multirow[t]{2}{*}{$0.22395 \mathrm{e}-34$} & $8 e \rightarrow 23 t_{2}$ & 49.52 \\
\hline & & & $22 t_{2} \rightarrow 11 e$ & 45.21 \\
\hline & \multirow[t]{3}{*}{10.493} & \multirow[t]{3}{*}{$0.40701 \mathrm{e}-34$} & $8 e \rightarrow 23 t_{2}$ & 38.19 \\
\hline & & & $21 t_{2} \rightarrow 10 e$ & 26.53 \\
\hline & & & $22 t_{2} \rightarrow 11 e$ & 20.88 \\
\hline & 10.500 & $0.50353 \mathrm{e}-35$ & $20 t_{2} \rightarrow 23 t_{2}$ & 88.07 \\
\hline & 10.641 & $0.21616 \mathrm{e}-39$ & $8 t_{1} \rightarrow 10 e$ & 90.58 \\
\hline & 10.698 & $0.11680 \mathrm{e}-37$ & $22 t_{2} \rightarrow 26 t_{2}$ & 98.56 \\
\hline & 10.904 & $0.99746 \mathrm{e}-34$ & $7 t_{1} \rightarrow 10 e$ & 98.27 \\
\hline & \multirow[t]{2}{*}{10.959} & \multirow[t]{2}{*}{$0.20640 \mathrm{e}-35$} & $9 e \rightarrow 24 t_{2}$ & 63.73 \\
\hline & & & $21 t_{2} \rightarrow 9 t_{1}$ & 33.28 \\
\hline & \multirow[t]{2}{*}{10.980} & \multirow[t]{2}{*}{$0.22762 \mathrm{e}-35$} & $21 t_{2} \rightarrow 9 t_{1}$ & 52.53 \\
\hline & & & $9 e \rightarrow 24 t_{2}$ & 34.95 \\
\hline & 11.027 & $0.31398 \mathrm{e}-34$ & $20 t_{2} \rightarrow 10 e$ & 96.78 \\
\hline & \multirow[t]{2}{*}{11.184} & \multirow[t]{2}{*}{$0.24337 \mathrm{e}-35$} & $8 t_{1} \rightarrow 9 t_{1}$ & 77.16 \\
\hline & & & $14 a_{1} \rightarrow 9 t_{1}$ & 11.39 \\
\hline & 11.186 & $0.51450 \mathrm{e}-38$ & $22 t_{2} \rightarrow 10 t_{1}$ & 91.04 \\
\hline & 11.248 & $0.32106 \mathrm{e}-35$ & $8 t_{1} \rightarrow 15 a_{1}$ & 96.90 \\
\hline & \multirow[t]{3}{*}{11.294} & \multirow[t]{3}{*}{$0.64323 \mathrm{e}-36$} & $14 a_{1} \rightarrow 9 t_{1}$ & 55.04 \\
\hline & & & $22 t_{2} \rightarrow 27 t_{2}$ & 18.10 \\
\hline & & & $6 t_{1} \rightarrow 23 t_{2}$ & 10.63 \\
\hline & 11.388 & $0.10163 \mathrm{e}-33$ & $7 e \rightarrow 23 t_{2}$ & 28.53 \\
\hline
\end{tabular}


Table S5: TDDFT (SR-SAOP/TZP//SR-LDA/TZP) dipole forbidden transitions

\begin{tabular}{ccccc} 
Symmetry & $\mathrm{E}(\mathrm{eV}) \mathrm{f}$ & Assignment & $\%$ \\
\hline & & $22 t_{2} \rightarrow 27 t_{2}$ & 40.94 \\
& & $6 t_{1} \rightarrow 23 t_{2}$ & 26.29 \\
11.403 & $0.31738 \mathrm{e}-33$ & $7 e \rightarrow 23 t_{2}$ & 65.31 \\
& & $22 t_{2} \rightarrow 27 t_{2}$ & 23.51 \\
11.419 & $0.55380 \mathrm{e}-35$ & $7 t_{1} \rightarrow 9 t_{1}$ & 47.20 \\
\hline \hline
\end{tabular}

Table S6: TDDFT (SR-SAOP/TZP//SR-LDA/TZP) singlet-triplet transitions, their symmetry label, the corresponding oscillator strenghts (f), and description of the dominant one-electron excitations (contributions greater than $10 \%$ ) of $\operatorname{Pt}\left(\mathrm{PF}_{3}\right)_{4}$

\begin{tabular}{|c|c|c|c|c|}
\hline Symmetry & $\mathrm{E}(\mathrm{eV})$ & $\mathrm{f}$ & Assignment & $\%$ \\
\hline \multirow[t]{13}{*}{$A_{1}$} & 5.3835 & 0.0000 & $22 t_{2} \rightarrow 23 t_{2}$ & 99.72 \\
\hline & 8.2005 & 0.0000 & $22 t_{2} \rightarrow 24 t_{2}$ & 99.72 \\
\hline & 8.5016 & 0.0000 & $9 e \rightarrow 10 e$ & 98.40 \\
\hline & 9.5889 & 0.0000 & $22 t_{2} \rightarrow 25 t_{2}$ & 99.42 \\
\hline & 9.7101 & 0.0000 & $21 t_{2} \rightarrow 23 t_{2}$ & 98.08 \\
\hline & 10.402 & 0.0000 & $20 t_{2} \rightarrow 23 t_{2}$ & 97.77 \\
\hline & 10.598 & 0.0000 & $22 t_{2} \rightarrow 26 t_{2}$ & 98.59 \\
\hline & 10.938 & 0.0000 & $8 e \rightarrow 10 e$ & 96.71 \\
\hline & 11.099 & 0.0000 & $8 t_{1} \rightarrow 9 t_{1}$ & 99.98 \\
\hline & 11.212 & 0.0000 & $14 a_{1} \rightarrow 15 a_{1}$ & 93.55 \\
\hline & 11.305 & 0.0000 & $22 t_{2} \rightarrow 27 t_{2}$ & 87.42 \\
\hline & 11.345 & 0.0000 & $7 t_{1} \rightarrow 9 t_{1}$ & 82.80 \\
\hline & & & $19 t_{2} \rightarrow 23 t_{2}$ & 14.41 \\
\hline$A_{2}$ & 6.6120 & 0.0000 & $22 t_{2} \rightarrow 9 t_{1}$ & 100.00 \\
\hline
\end{tabular}


Table S6: TDDFT (SR-SAOP/TZP//SR-LDA/TZP) singlet-triplet transitions

\begin{tabular}{|c|c|c|c|c|}
\hline Symmetry & $\mathrm{E}(\mathrm{eV})$ & $\mathrm{f}$ & Assignment & $\%$ \\
\hline & 8.7859 & 0.0000 & $9 e \rightarrow 10 e$ & 100.00 \\
\hline & 10.021 & 0.0000 & $8 t_{1} \rightarrow 23 t_{2}$ & 99.73 \\
\hline & 10.346 & 0.0000 & $7 t_{1} \rightarrow 23 t_{2}$ & 99.79 \\
\hline & 10.928 & 0.0000 & $21 t_{2} \rightarrow 9 t_{1}$ & 99.68 \\
\hline & 10.979 & 0.0000 & $8 e \rightarrow 10 e$ & 99.18 \\
\hline & 11.167 & 0.0000 & $1 a_{2} \rightarrow 15 a_{1}$ & 94.83 \\
\hline & 11.186 & 0.0000 & $22 t_{2} \rightarrow 10 t_{1}$ & 99.53 \\
\hline & 11.316 & 0.0000 & $6 t_{1} \rightarrow 23 t_{2}$ & 95.31 \\
\hline$E$ & 5.4175 & 0.0000 & $22 t_{2} \rightarrow 23 t_{2}$ & 99.68 \\
\hline & 6.4855 & 0.0000 & $22 t_{2} \rightarrow 9 t_{1}$ & 99.58 \\
\hline & 8.2430 & 0.0000 & $22 t_{2} \rightarrow 24 t_{2}$ & 99.95 \\
\hline & 8.6493 & 0.0000 & $9 e \rightarrow 10 e$ & 99.54 \\
\hline & 9.3530 & 0.0000 & $9 e \rightarrow 15 a_{1}$ & 99.03 \\
\hline & 9.5944 & 0.0000 & $22 t_{2} \rightarrow 25 t_{2}$ & 96.66 \\
\hline & 9.7641 & 0.0000 & $21 t_{2} \rightarrow 23 t_{2}$ & 95.56 \\
\hline & 9.9994 & 0.0000 & $8 t_{1} \rightarrow 23 t_{2}$ & 98.95 \\
\hline & 10.338 & 0.0000 & $7 t_{1} \rightarrow 23 t_{2}$ & 99.42 \\
\hline & 10.434 & 0.0000 & $20 t_{2} \rightarrow 23 t_{2}$ & 98.57 \\
\hline & 10.520 & 0.0000 & $1 a_{2} \rightarrow 10 e$ & 97.98 \\
\hline & 10.563 & 0.0000 & $14 a_{1} \rightarrow 10 e$ & 93.00 \\
\hline & 10.659 & 0.0000 & $22 t_{2} \rightarrow 26 t_{2}$ & 92.49 \\
\hline & 10.739 & 0.0000 & $9 e \rightarrow 16 a_{1}$ & 92.86 \\
\hline & 10.857 & 0.0000 & $21 t_{2} \rightarrow 9 t_{1}$ & 94.78 \\
\hline & 10.959 & 0.0000 & $8 e \rightarrow 10 e$ & 98.66 \\
\hline & 11.046 & 0.0000 & $8 t_{1} \rightarrow 9 t_{1}$ & 97.84 \\
\hline
\end{tabular}


Table S6: TDDFT (SR-SAOP/TZP//SR-LDA/TZP) singlet-triplet transitions

\begin{tabular}{|c|c|c|c|c|}
\hline \multirow[t]{5}{*}{ Symmetry } & $\mathrm{E}(\mathrm{eV})$ & $\mathrm{f}$ & Assignment & $\%$ \\
\hline & 11.172 & 0.0000 & $22 t_{2} \rightarrow 10 t_{1}$ & 99.63 \\
\hline & 11.320 & 0.0000 & $6 t_{1} \rightarrow 23 t_{2}$ & 84.38 \\
\hline & & & $19 t_{2} \rightarrow 23 t_{2}$ & 10.93 \\
\hline & 11.335 & 0.0000 & $22 t_{2} \rightarrow 27 t_{2}$ & 96.19 \\
\hline \multirow[t]{21}{*}{$T_{1}$} & 5.4149 & 0.0000 & $22 t_{2} \rightarrow 23 t_{2}$ & 97.81 \\
\hline & 6.0224 & 0.0000 & $22 t_{2} \rightarrow 10 e$ & 98.39 \\
\hline & 6.5170 & 0.0000 & $22 t_{2} \rightarrow 9 t_{1}$ & 98.89 \\
\hline & 8.1942 & 0.0000 & $9 e \rightarrow 23 t_{2}$ & 99.71 \\
\hline & 8.2545 & 0.0000 & $22 t_{2} \rightarrow 24 t_{2}$ & 99.93 \\
\hline & 9.2941 & 0.0000 & $9 e \rightarrow 9 t_{1}$ & 99.80 \\
\hline & 9.6031 & 0.0000 & $22 t_{2} \rightarrow 25 t_{2}$ & 98.53 \\
\hline & 9.8198 & 0.0000 & $21 t_{2} \rightarrow 23 t_{2}$ & 97.56 \\
\hline & 9.9892 & 0.0000 & $1 a_{2} \rightarrow 23 t_{2}$ & 85.98 \\
\hline & & & $8 t_{1} \rightarrow 23 t_{2}$ & 13.10 \\
\hline & 10.018 & 0.0000 & $8 t_{1} \rightarrow 23 t_{2}$ & 86.40 \\
\hline & & & $1 a_{2} \rightarrow 23 t_{2}$ & 13.32 \\
\hline & 10.321 & 0.0000 & $7 t_{1} \rightarrow 23 t_{2}$ & 98.68 \\
\hline & 10.340 & 0.0000 & $21 t_{2} \rightarrow 10 e$ & 95.66 \\
\hline & 10.437 & 0.0000 & $22 t_{2} \rightarrow 11 e$ & 85.65 \\
\hline & & & $8 e \rightarrow 23 t_{2}$ & 10.05 \\
\hline & 10.445 & 0.0000 & $8 e \rightarrow 23 t_{2}$ & 85.53 \\
\hline & & & $22 t_{2} \rightarrow 11 e$ & 12.00 \\
\hline & 10.459 & 0.0000 & $20 t_{2} \rightarrow 23 t_{2}$ & 95.67 \\
\hline & 10.534 & 0.0000 & $8 t_{1} \rightarrow 10 e$ & 97.47 \\
\hline & 10.677 & 0.0000 & $22 t_{2} \rightarrow 26 t_{2}$ & 99.07 \\
\hline
\end{tabular}


Table S6: TDDFT (SR-SAOP/TZP//SR-LDA/TZP) singlet-triplet transitions

\begin{tabular}{|c|c|c|c|c|}
\hline Symmetry & $\mathrm{E}(\mathrm{eV})$ & $\mathrm{f}$ & Assignment & $\%$ \\
\hline & 10.864 & 0.0000 & $7 t_{1} \rightarrow 10 e$ & 87.06 \\
\hline & \multirow[t]{2}{*}{10.882} & \multirow[t]{2}{*}{0.0000} & $21 t_{2} \rightarrow 9 t_{1}$ & 79.62 \\
\hline & & & $7 t_{1} \rightarrow 10 e$ & 11.98 \\
\hline & 10.949 & 0.0000 & $9 e \rightarrow 24 t_{2}$ & 97.94 \\
\hline & 10.984 & 0.0000 & $20 t_{2} \rightarrow 10 e$ & 97.78 \\
\hline & 11.045 & 0.0000 & $8 t_{1} \rightarrow 9 t_{1}$ & 97.35 \\
\hline & 11.091 & 0.0000 & $14 a_{1} \rightarrow 9 t_{1}$ & 91.39 \\
\hline & \multirow[t]{2}{*}{11.180} & \multirow[t]{2}{*}{0.0000} & $22 t_{2} \rightarrow 10 t_{1}$ & 85.14 \\
\hline & & & $8 t_{1} \rightarrow 15 a_{1}$ & 12.87 \\
\hline & \multirow[t]{2}{*}{11.181} & \multirow[t]{2}{*}{0.0000} & $8 t_{1} \rightarrow 15 a_{1}$ & 82.49 \\
\hline & & & $22 t_{2} \rightarrow 10 t_{1}$ & 13.45 \\
\hline & \multirow[t]{2}{*}{11.306} & \multirow[t]{2}{*}{0.0000} & $6 t_{1} \rightarrow 23 t_{2}$ & 84.62 \\
\hline & & & $7 e \rightarrow 23 t_{2}$ & 10.32 \\
\hline & \multirow[t]{2}{*}{11.329} & \multirow[t]{2}{*}{0.0000} & $7 e \rightarrow 23 t_{2}$ & 78.71 \\
\hline & & & $6 t_{1} \rightarrow 23 t_{2}$ & 10.43 \\
\hline & \multirow[t]{2}{*}{11.361} & \multirow[t]{2}{*}{0.0000} & $19 t_{2} \rightarrow 23 t_{2}$ & 75.78 \\
\hline & & & $22 t_{2} \rightarrow 27 t_{2}$ & 15.92 \\
\hline \multirow[t]{8}{*}{$T_{2}$} & 5.4183 & 0.0000 & $22 t_{2} \rightarrow 23 t_{2}$ & 99.25 \\
\hline & 5.9203 & 0.0000 & $22 t_{2} \rightarrow 10 e$ & 97.97 \\
\hline & 6.4289 & 0.0000 & $22 t_{2} \rightarrow 9 t_{1}$ & 98.37 \\
\hline & 6.6579 & 0.0000 & $22 t_{2} \rightarrow 15 a_{1}$ & 99.58 \\
\hline & 7.9813 & 0.0000 & $22 t_{2} \rightarrow 16 a_{1}$ & 97.85 \\
\hline & 8.1576 & 0.0000 & $9 e \rightarrow 23 t_{2}$ & 98.65 \\
\hline & 8.2282 & 0.0000 & $22 t_{2} \rightarrow 24 t_{2}$ & 99.13 \\
\hline & 9.2594 & 0.0000 & $9 e \rightarrow 9 t_{1}$ & 99.74 \\
\hline
\end{tabular}


Table S6: TDDFT (SR-SAOP/TZP//SR-LDA/TZP) singlet-triplet transitions

\begin{tabular}{|c|c|c|c|c|}
\hline Symmetry & $\mathrm{E}(\mathrm{eV})$ & $\mathrm{f}$ & Assignment & $\%$ \\
\hline & 9.5997 & 0.0000 & $22 t_{2} \rightarrow 25 t_{2}$ & 99.54 \\
\hline & 9.7810 & 0.0000 & $21 t_{2} \rightarrow 23 t_{2}$ & 98.93 \\
\hline & 9.9997 & 0.0000 & $8 t_{1} \rightarrow 23 t_{2}$ & 96.03 \\
\hline & 10.013 & 0.0000 & $14 a_{1} \rightarrow 23 t_{2}$ & 93.22 \\
\hline & 10.309 & 0.0000 & $7 t_{1} \rightarrow 23 t_{2}$ & 94.73 \\
\hline & 10.339 & 0.0000 & $21 t_{2} \rightarrow 10 e$ & 91.56 \\
\hline & 10.411 & 0.0000 & $8 e \rightarrow 23 t_{2}$ & 92.05 \\
\hline & 10.420 & 0.0000 & $22 t_{2} \rightarrow 11 e$ & 73.29 \\
\hline & & & $20 t_{2} \rightarrow 23 t_{2}$ & 17.55 \\
\hline & 10.446 & 0.0000 & $20 t_{2} \rightarrow 23 t_{2}$ & 77.35 \\
\hline & & & $22 t_{2} \rightarrow 11 e$ & 18.61 \\
\hline & 10.468 & 0.0000 & $22 t_{2} \rightarrow 17 a_{1}$ & 97.15 \\
\hline & 10.534 & 0.0000 & $8 t_{1} \rightarrow 10 e$ & 97.98 \\
\hline & 10.637 & 0.0000 & $22 t_{2} \rightarrow 26 t_{2}$ & 99.06 \\
\hline & 10.837 & 0.0000 & $21 t_{2} \rightarrow 9 t_{1}$ & 77.20 \\
\hline & & & $7 t_{1} \rightarrow 10 e$ & 18.38 \\
\hline & 10.855 & 0.0000 & $7 t_{1} \rightarrow 10 e$ & 79.59 \\
\hline & & & $21 t_{2} \rightarrow 9 t_{1}$ & 18.45 \\
\hline & 10.943 & 0.0000 & $9 e \rightarrow 24 t_{2}$ & 97.53 \\
\hline & 10.968 & 0.0000 & $20 t_{2} \rightarrow 10 e$ & 96.84 \\
\hline & 10.996 & 0.0000 & $21 t_{2} \rightarrow 15 a_{1}$ & 98.06 \\
\hline & 11.035 & 0.0000 & $1 a_{2} \rightarrow 9 t_{1}$ & 74.60 \\
\hline & & & $8 t_{1} \rightarrow 9 t_{1}$ & 22.99 \\
\hline & 11.095 & 0.0000 & $8 t_{1} \rightarrow 9 t_{1}$ & 76.12 \\
\hline & & & $1 a_{2} \rightarrow 9 t_{1}$ & 23.82 \\
\hline
\end{tabular}


Table S6: TDDFT (SR-SAOP/TZP//SR-LDA/TZP) singlet-triplet transitions

\begin{tabular}{ccccc} 
Symmetry & E $(\mathrm{eV})$ & $\mathrm{f}$ & Assignment & $\%$ \\
\hline 11.171 & 0.0000 & $22 t_{2} \rightarrow 10 t_{1}$ & 99.54 \\
11.316 & 0.0000 & $7 e \rightarrow 23 t_{2}$ & 75.52 \\
& & $6 t_{1} \rightarrow 23 t_{2}$ & 15.37 \\
11.320 & 0.0000 & $6 t_{1} \rightarrow 23 t_{2}$ & 74.34 \\
& & $19 t_{2} \rightarrow 23 t_{2}$ & 13.34 \\
11.346 & 0.0000 & $22 t_{2} \rightarrow 27 t_{2}$ & 89.45 \\
\hline \hline
\end{tabular}

\section{Importance of the relativistic effects and choice of the}

\section{functional}

Figure S1 shows the excitation spectra (oscillator strengths) calculated with four different methods at the SR-LDA/TZP ground state geometry. The comparison serves as demonstration how important is it to include the relativistic effects and to use a proper functional.

Relativistic TDDFT calculations at SR-SAOP/TZP level of theory are in excellent agreement with electron energy loss spectrum ${ }^{5}$ and is discussed in the main text. The inclusion of spin-orbit coupling changes the spectrum only marginally, the reasons for this explained in the main text. Position of the excited states in NR calculations is shifted towards lower energies. Inclusion of relativistic effects is also important for the oscillator strengths - NR calculations are not able to reproduce correctly relative position of the highest intensity peak. Calculations using general gradient approximation, e.g. PW91, Fig. S1 are not able to reproduce high-energy excitations. 



Figure S1: Top panel: Electron energy loss spectrum of $\mathrm{Pt}\left(\mathrm{PF}_{3}\right)_{4}$. Lower panels: TDDFT calculated transition energies at different levels of theory (SR-SAOP/TZP; the same with spin-orbit splitting included; NR-SAOP/TZP; NR-PW91); the heights of vertical lines indicate calculated oscillator strengths

\section{Excited states at different $\mathrm{Pt}-\mathrm{P}$ bond distances}

Table S7 lists calculated excited states at SR-BHandHLYP/TZP//SR-B3LYP-D3/TZP level of theory at two different $\mathrm{Pt}-\mathrm{P}$ bond lengths, at $2.266 \AA$ (equilibrium distance in $\mathrm{Pt}\left(\mathrm{PF}_{3}\right)_{4}$ at SR-B3LYP-D3/TZP level of theory) and at $10.000 \AA$ ( $\mathrm{PF}_{3}$ essentially dissociated). Symmetry labels of excited states in $C_{3 v}$ point group is given as well. These excited states correspond to those plotted in Fig. 2 in the main text.

The excited states from Table S7 are calculated at different level of theory than those listed in Table S4, as explained in the main text, and are shifted to higher energies for approx. $0.5 \mathrm{eV}$. All listed excited states at Pt-P bond length of $10.000 \AA$ exactly correspond to those of $\mathrm{Pt}\left(\mathrm{PF}_{3}\right)_{3}$ in its ground state energy minimum that are listed in Table $\mathrm{S}$. 
Table S7: TDDFT (SR-BHandHLYP/TZP//SR-B3LYP-D3/TZP) excitation energies $(\mathrm{eV})$ of $\mathrm{Pt}\left(\mathrm{PF}_{3}\right)_{4}$ on different $\mathrm{Pt}-\mathrm{P}$ bond distances and comparison to the excitation energies of $\mathrm{Pt}\left(\mathrm{PF}_{3}\right)_{3}$ and symmetry label of the states in $C_{3 v}$ point group

\begin{tabular}{l|ccc|ccc} 
& \multicolumn{5}{|c|}{ Singlets } & Triplets \\
& $\mathrm{Pt}\left(\mathrm{PF}_{3}\right)_{4}$ & $\mathrm{Pt}\left(\mathrm{PF}_{3}\right)_{3}--\mathrm{PF}_{3}$ & $\mathrm{Pt}\left(\mathrm{PF}_{3}\right)_{3}$ & $\mathrm{Pt}\left(\mathrm{PF}_{3}\right)_{4}$ & $\mathrm{Pt}\left(\mathrm{PF}_{3}\right)_{3}--\mathrm{PF}_{3}$ & $\mathrm{Pt}\left(\mathrm{PF}_{3}\right)_{3}$ \\
\hline$A_{1}$ & 2.266 & 10.000 & - & 2.266 & 10.000 & - \\
& 6.3899 & 5.5861 & 5.5912 & 5.46119 & 4.5323 & 4.5365 \\
& 6.6648 & 8.0179 & 8.0219 & 5.64451 & 6.8357 & 6.8400 \\
& 7.0558 & 8.6682 & 8.6792 & 6.23193 & 7.8420 & 7.8518 \\
& 7.7879 & 8.7361 & 8.7465 & 7.15288 & 8.2036 & 8.2173 \\
& 8.1491 & 8.7758 & 8.7832 & 7.44466 & 8.4792 & 8.4858 \\
\hline \multirow{2}{*}{$E$} & 6.0946 & 7.6079 & 7.6115 & 5.55630 & 6.9189 & 6.9218 \\
& 6.6925 & 8.3972 & 8.4073 & 6.49748 & 8.1073 & 8.1159 \\
& 7.8192 & 8.5985 & 8.6046 & 7.47542 & 8.4896 & 8.4964 \\
& 7.8363 & 8.7215 & 8.7207 & 7.83484 & 8.7117 & 8.7110 \\
& 8.4011 & 9.5425 & 9.5470 & 8.28981 & 9.5499 & 9.5546 \\
\hline \multirow{2}{*}{$E$} & 6.0946 & 5.0003 & 5.0062 & 5.55630 & 4.5651 & 4.5698 \\
& 6.2780 & 6.3020 & 6.3098 & 5.64451 & 5.7272 & 5.7350 \\
& 6.3899 & 7.8224 & 7.8256 & 5.65921 & 6.9881 & 6.9915 \\
& 6.6925 & 8.0349 & 8.0392 & 6.23193 & 7.4442 & 7.4444 \\
& 7.0558 & 8.4355 & 8.4400 & 6.49748 & 7.6280 & 7.6320 \\
& 7.7879 & 8.5087 & 8.5182 & 7.15288 & 7.8906 & 7.9000 \\
& 7.8192 & 8.7714 & 8.7804 & 7.29160 & 8.0153 & 8.0260 \\
& 7.9490 & 8.8941 & 8.9010 & 7.44466 & 8.3955 & 8.4024 \\
& 8.1491 & 8.9364 & 8.9411 & 7.47542 & 8.6720 & 8.6820 \\
& 8.4011 & 8.9854 & 8.9966 & 8.11793 & 8.8854 & 8.8911 \\
\hline
\end{tabular}

\section{References}

(1) Drews, T.; Rusch, D.; Seidel, S.; Willemsen, S.; Seppelt, K. Systematic Reactions of [Pt $\left.\left(\mathrm{PF}_{3}\right)_{4}\right]$. Chem. Eur. J. 2008, 14, 4280-4286.

(2) Hu, P.; Luo, Q.; shu Li, Q.; Xie, Y.; King, R. B.; Schaefer, H. F. Construction of the Tetrahedral Trifluorophosphine Platinum Cluster $\mathrm{Pt}_{4}\left(\mathrm{PF}_{3}\right)_{8}$ from Smaller Building Blocks. Inorg. Chem. 2014, 53, 5300-5310.

(3) Ritz, C. L.; Bartell, L. S. Molecular structure of $\mathrm{Pt}\left(\mathrm{PF}_{3}\right)_{4}$ by gas-phase electron diffraction. J. Mol. Structure 1976, 31, 73-76. 
(4) Bligh-Smith, R. M.; Edwards, H. G. M.; Fawcett, V. Force-Constant Calculations for $\mathrm{Ni}\left(\mathrm{PF}_{3}\right)_{4}, \mathrm{Pd}\left(\mathrm{PF}_{3}\right)_{4}$ and $\mathrm{Pt}\left(\mathrm{PF}_{3}\right)_{4}$. Spectrochim. Acta A 1987, 43, 1069-1073.

(5) Allan, M. Electron Scattering in $\mathrm{Pt}\left(\mathrm{PF}_{3}\right)_{4}$ : Elastic Scattering, Vibrational, and Electronic Excitation. J. Chem. Phys. 2011, 134, 204309. 